\title{
Photographies : un album à double préface
}

Joëlle Gleize et David Zemmour

\section{(2) OpenEdition}

\section{Journals}

Édition électronique

URL : https://journals.openedition.org/ccs/2711

DOI : $10.4000 /$ ccs.2711

ISSN : 2558-782X

\section{Éditeur :}

Presses universitaires de Rennes, Association des lecteurs de Claude Simon

\section{Édition imprimée}

Date de publication : 3 septembre 2020

Pagination : 33-36

ISBN : 978-2-7535-8065-7

ISSN : 1774-9425

\section{Référence électronique}

Joëlle Gleize et David Zemmour, «Photographies : un album à double préface », Cahiers Claude Simon [En ligne], 15 | 2020, mis en ligne le 03 septembre 2021, consulté le 05 décembre 2022. URL : http:// journals.openedition.org/ccs/2711; DOI : https://doi.org/10.4000/ccs.2711 


\title{
PHOTOGRAPHIES : UN ALBUM À DOUBLE PRÉFACE
}

\author{
Joëlle GLEIZE et David ZEMMOUR
}

Tardivement, à un moment où son œuvre romanesque est pleinement reconnue, Simon révèle enfin la part photographique de son œuvre, dans son Album d'un amateur ${ }^{1}$ d'abord, paru en 1988 et resté confidentiel, puis dans l'album Photographies ${ }^{2}$ en 1992, une part visuelle qui a précédé et accompagné ses premiers livres. Contrairement à l'œuvre picturale, l'œuvre photographique a été préservée de la destruction et est alors exposée ${ }^{3}$, puis en partie au moins, publiée. Photographies, en 1992, avec son grand format, la qualité des reproductions pleine page en noir et blanc, donne le statut d'œuvre visuelle à part entière à ces images.

L'œuvre est doublement préfacée, par Denis Roche d'abord, par Claude Simon ensuite ${ }^{4}$.

Claude Simon évoque sobrement sa pratique, en évitant tout propos théorique mais en soulignant ce qui fait pour lui la singularité fascinante de cet art. Il part d'une définition aussi simple qu'évidente (" photographier, c'est fixer au moyen d'une image quelque chose qui s'est produit à un certain endroit et à un certain moment ») pour constater que la photographie entretient avec la peinture et la littérature des liens étroits. À maints égards, les préoc-

1. Album d'un amateur est paru en édition à tirage limité (999 ex.) en Allemagne chez Remagen-Rolandseck, Rommerskirchen, en 1988.

2. Photographies 1937-1970, paraît dans la collection Photo-Cinéma chez Maeght en mars 1992.

3. La galerie Maeght expose les photographies de l'album à Paris en mars 1992; une exposition d'autres photographies de C. Simon est organisée à la galerie du Château-d'Eau, à Toulouse (9 avril-4 mai 1992).

4. Phot., resp. p. 6-13 et 15-17. 
cupations de l'artiste sont les mêmes. Claude Simon s'intéresse à la photographie en peintre et, dans les deux cas, « ce qui retient d'abord [s] on attention et suscite [s] on plaisir, ce sont les qualités proprement picturales de ces tableaux, c'est-à-dire les accords de couleurs que l'artiste a su trouver, l'équilibre et l'harmonie des formes (et des vides) qu'il a délimités». En outre, comme la peinture et la littérature, la photographie interroge la relation complexe qui peut exister entre l'œuvre et la part de réel qui l'a suscitée. C'est là tout le débat sur la place et le rôle du référent, occasion pour Simon de rappeler, sans trop y insister, la position aussi hégémoniste que radicalement textualiste de Jean Ricardou, en particulier lors du colloque de Cerisy consacré au Nouveau Roman en 1971, lorsque Simon fit état de documents liés à son œuvre, en l'occurrence " la lettre d'un colonel de cavalerie confirmant l'exactitude "jusque dans ses moindres détails" d'un épisode de La Route des Flandres et [...] un billet de banque chilien décrit dans Les Corps conducteurs ${ }^{5}$ ". Enfin ces trois arts ont en commun de relever d'une démarche laborieuse et artisanale, faite d'essais, d'expériences, d'ajouts ou de suppressions : « [p] as plus en photographiant qu'en écrivant je n'ai le don de spontanéité, de sorte que, me méfiant de moi-même, il m'arrive le plus souvent [...] de cadrer mon "sujet" très largement, sachant qu'au calme de la chambre noire, sans hâte et en toute tranquillité, je pourrai toujours supprimer ce qui me semble inutile, risque de disperser l'attention ou de déséquilibrer la composition ».

Mais les modalités de cet artisanat ne sont pas identiques, et c'est là la première singularité de la photographie qui, une fois la photo prise, laisse assez peu de marge de manœuvre : « je ne dispose pour corriger mes brouillons photographiques que du choix entre les gradations de divers papiers plus ou moins contrastés et de la possibilité d'y retrancher, jamais d'y ajouter ». L'autre singularité, essentielle, concerne le rapport spécifique de la photo au temps. En effet, "[a]ucun esprit humain ne peut garder en mémoire ce qu'embrasse le regard pendant une de ces incessantes fractions de seconde que le temps fait se succéder à une vitesse tellement vertigineuse que lorsque je trace la dernière lettre d'un mot le geste de ma main dessinant la précédente

5. «Les lettres d'un officier de cavalerie, je dois avouer qu'elles m'importent assez peu. [...] Ce qui est donné par Simon, ce sont les référents de la fiction : cela ne veut nullement dire que la fiction obtenue par le texte est l'équivalent du référent donné à titre documentaire " (Jean Ricardou lors de la discussion suivant sa communication "Le Nouveau Roman existe-t-il? ", dans J. Ricardou et F. Van Rossum-Guyon (dir.), Nouveau Roman : hier, aujourd'hui, Hermann, 2011, t. 1 ("Problèmes généraux »), p. 29-30. Pour une contextualisation de ces échanges, voir Anthony C. Pugh, "Claude Simon et la route de la référence », Revue des Sciences humaines, $\mathrm{n}^{\circ} 220,1990$, p. 23-45. 
appartient déjà au passé ». La photo, elle, permet de saisir au vol ce présent insaisissable; elle seule possède le pouvoir « de fixer, de mémoriser ce que notre mémoire elle-même est incapable de retenir, c'est-à-dire l'image de quelque chose qui n'a eu lieu, n'a existé, que dans une fraction infime de temps ». Une œuvre peinte est toujours le résultat des regards successifs portés par l'artiste sur le modèle. Elle porte toujours la trace de son élaboration, que celle-ci ait requis quelques heures ou plusieurs jours. Et Claude Simon de conclure : "Qu'il s'agisse de Cézanne exigeant d'innombrables séances de pose ou de Van Gogh capable d'exécuter une toile en une matinée ou un après-midi, toute production d'image s'élabore dans une durée, est le résultat d'une méditation, d'une addition et d'une combinaison de présents accumulés [...], et seule, à ma connaissance du moins, la photographie peut saisir et garder une trace de ce qui n'avait encore jamais été et ne sera plus jamais. Je me demande si, en définitive et au-delà de toute autre considération, ce n'est pas l'attrait de ce pouvoir quelque peu magique qui m’a poussé à m'y essayer ".

Intitulée " De la ténèbre inverse ", l'autre préface est signée d'un écrivain qui est aussi photographe et éditeur. C'est avec Denis Roche l'éditeur que Simon est entré en relation en 1986 à l'occasion de la publication, dans la collection du Seuil qu'il dirige, de l'étude de Lucien Dällenbach qui lui est consacrée $^{6}$. Des liens d'amitié se nouent alors entre les deux écrivains, et leur intérêt commun pour la photographie y contribue certainement. Simon admire les images de Denis Roche ${ }^{7}$; ces affinités et leurs échanges autour de la photographie l'amènent à se tourner vers lui pour préfacer l'album de 1992. "De la ténèbre inverse " est une préface elle aussi peu théorique, une préface d'écrivain et de photographe. C'est en praticien que Roche évoque le " piétinement " du photographe à l'affût et qu'il décrit le cadrage et le recadrage par lesquels Simon "ferme " l'image; c'est en écrivain et en photographe qu'il interroge le passage de la photographie vers l'écriture, de l'extérieur vers l'intérieur, et qu'il rapproche les deux pratiques en une analyse remarquable de l'écriture du romancier : " "fermant" l'objectif ou l'"ouvrant" jusqu'à faire entrer tant de choses d'un seul coup dans un étrange mouvement effaré de la lumière; puis émiettant, comme on fait d'une feuille sèche entre deux doigts,

6. L. Dällenbach, Claude Simon, coll. «Les contemporains », Seuil, 1988. Denis Roche choisit pour lancer sa collection trois auteurs à qui il voue une grande admiration : Francis Ponge, Georges Perec et Claude Simon.

7. Dans une lettre à Denis Roche du 21 octobre 1989, Claude Simon souhaite voir publier un album des images de Roche, et dit aimer particulièrement l'" Hommage à Henry Moore » de Roche, qu'il qualifie de " génial " (voir cet " hommage " dans le cahier iconographique, p. xxIv). 
fragmentant ce qu'il voit, le dispersant, gagné par le lyrisme du diminuendo ", l'écrivain comme le photographe luttant pour " rejeter [le]noir et [la ténèbre] dans le hors champ, qu'il n'en subsiste que la trace ".

De ces deux textes en étroite complémentarité, nous ne pouvons offrir la lecture que de celui de Denis Roche, avec le regret de ne pouvoir mieux servir la pensée photographique de Claude Simon ${ }^{8}$. Alors même qu'il ne revendique pas le statut d'artiste, celui-ci n'en offre pas moins, avec Photographies, un album où la composition de chaque image, son harmonie et sa rigueur formelle aussi bien que la structuration du volume sont bien d'un photographe. Et quelles que soient les nombreuses interactions - indéniables et passionnantes - entre les deux versants inégaux de l'œuvre, l'élaboration esthétique de chaque image comme celle de leur succession sont telles que l'œuvre photographique, quoique seconde, n'a nul besoin d'être rapportée à l'œuvre romanesque pour faire sens et valeur'.

8. Sur les difficultés rencontrées auprès de l'ayant-droit pour l'œuvre de Claude Simon, voir supra notre avant-propos, p. 13-17.

9. Plusieurs études remarquables sont consacrées à cette part de l'œuvre simonienne, comme en témoigne la synthèse bibliographique que Christine Genin propose dans ce numéro (p. 39-80). Il suffit à propos de Photographies de mentionner l'article inaugural de Georges Raillard, "Photographies de Claude Simon », La Quinzaine littéraire, 16-31 mars 1992, p. 14-15; puis celui de Jean H. Duffy, "Claude Simon's Photographies: a Formal and Thematic Analysis ", Nottingham French Studies, 33, 2, 1994, p. 65-77, traduit sous le titre "Analyse formelle et thématique de Photographies de Claude Simon ", La Licorne, $\mathrm{n}^{\circ} 71$ ("Les Images chez Claude Simon : des mots pour le voir »), 2004, p. 133-151; celui de Philippe Ortel, "Le réseau des instants : Photographies de Claude Simon, ibid., p. 153-168; ainsi que l'ouvrage d'Irene Albers, Claude Simon, moments photographiques, paru en allemand en 2002 avant d'être traduit en 2004. 